\title{
Patogênese, sinais clínicos e epidemiologia das intoxicações por plantas cianogênicas no nordeste brasileiro
}

\section{Pathogenesis, clinical signs and epidemiology of the poisoning by cyanogenic plants in the Brazilian northwest}

\author{
Antônio Carlos Lopes Câmara ${ }^{1}$; Luciana Dalcin²; Benito Soto-Blanco ${ }^{3 *}$
}

\section{Resumo}

O presente artigo objetiva revisar os mecanismos de ação das plantas cianogênicas e, adicionalmente, se inclui uma seção sobre sinais clínicos e distribuição dos surtos no Nordeste brasileiro. As plantas cianogênicas com registro de surtos de intoxicação são Anadenanthera colubrina var. cebil (Piptadenia macrocarpa), Cnidoscolus quercifolias (C. phyllacanthus), M. carthaginensis var glaziovii, Passiflora foetida, Piptadenia viriflora, Sorghum halepense e S. bicolor. É notória a grande distribuição e importância da intoxicação por plantas cianogênicas no Nordeste brasileiro, sendo primordial a divulgação e disseminação das informações dos surtos de intoxicação nos municípios com pouco conhecimento das espécies causadoras de intoxicação cianídrica.

Palavras-chave: Anadenanthera colubrina var. cebil, Manihot spp, Sorghum spp, plantas cianogênicas

\begin{abstract}
This review highlights the mechanisms of action of cyanogenic plants and provides clinical signs and distribution of outbreaks in Northeastern Brazil. Cyanogenic plants causing reported outbreaks of poisoning are Anadenanthera colubrina var. cebil (Piptadenia macrocarpa), Cnidoscolus quercifolias (C. phyllacanthus), M. carthaginensis var glaziovii, Passiflora foetida, Piptadenia viriflora, Sorghum halepense and S. bicolor. Findings summarized herein emphasize the great importance and wide distribution of cyanogenic plant poisoning in the Northeastern Brazil. Displaying information of animal poisoning outbreaks in cities with little knowledge of the species causing cyanogenic poisoning is primordial as a preventive measure.
\end{abstract}

Key words: Anadenanthera colubrina var. cebil, Manihot spp, Sorghum spp, cyanogenic plants

${ }^{1}$ Discente de Doutorado do Programa de Pós-graduação em Ciência Animal, Universidade Federal Rural do Semiárido, UFERSA, Mossoró, RN. E-mail: aclcamara@yahoo.com.br

2 Médica Veterinária do Hospital Veterinário, Universidade de Brasília, UnB, Brasília, DF. E-mail: dalcinvet@yahoo.com.br

${ }^{3}$ Prof. Dr., Dept ${ }^{\circ}$ de Clínica e Cirurgia Veterinárias, Universidade Federal de Minas Gerais, UFMG, Belo Horizonte, MG. E-mail: benito.blanco@pq.cnpq.br

* Autor para correspondência 


\section{Introdução}

De maneira geral, são denominadas plantas cianogênicas, aquelas que contém glicosídeos cianogênicos, sendo tais substâncias constantemente encontradas em plantas de muitas famílias, sobretudo as rosáceas, leguminosas, gramíneas e aráceas, mas também com frequência em passifloráceas, euforbiáceas e compostas (TOKARNIA; DÖBEREINER; VARGAS, 2002). Esses glicosídeos também têm sido observados em samambaias, cogumelos, fungos e bactérias. Ao todo, já se contam mais de 1000 espécies de vegetais cianogênicos distribuídas em 250 gêneros e 80 famílias (TOKARNIA et al., 2012).

Atualmente, é notório o crescente interesse pelo conhecimento da epidemiologia e distribuição das intoxicações por plantas nas diferentes regiões brasileiras, como observado em levantamentos na região do Seridó (SILVA et al., 2006) e mesorregiões Central e Oeste do Rio Grande do Norte (GERALDO NETO; SAKAMOTO; SOTOBLANCO, 2013), Sertão da Paraíba (ASSIS et al., 2009, 2010), Cariri cearense (BEZERRA et al., 2012), Norte piauiense (MELLO et al., 2010), Norte do Tocantins (DANTAS et al., 2011a, 2011b) e região Central de Rondônia (SCHONS et al., 2012). Nestes trabalhos é marcante o registro de surtos envolvendo plantas cianogênicas, incluindo Manihot spp., especialmente $M$. carthaginensis var glaziovii (maniçoba); Anadenanthera colubrina var. cebil (Piptadenia macrocarpa) (angico preto), Cnidoscolus quercifolias (C. phyllacanthus) (favela ou faveleira), Sorghum halepense (capim-de-boi ou sorgo de alepo) e S. bicolor (sorgo). Além destas, existem ainda Piptadenia viriflora (surucucu ou espinheiro) e Passiflora foetida (maracujá-domato ou canapú-fedorento) consideradas também como plantas cianogênicas de interesse pecuário no Nordeste brasileiro (TOKARNIA et al., 1999; CARVALHO et al., 2011; RIET-CORREA; BEZERRA; MEDEIROS, 2011; TOKARNIA et al., 2012). Deste modo, o presente trabalho objetiva revisar os mecanismos de ação das plantas cianogênicas e, adicionalmente, se inclui uma seção sobre sinais clínicos e distribuição dos surtos no Nordeste brasileiro, região esta com maior incidência de intoxicações.

\section{Mecanismo de Ação}

Dois grupos de produtos do metabolismo da planta, os glicosídeos cianogênicos e os cianolipídios, liberam ácido cianídrico por meio da ação de hidroxilases, sendo os glicosídeos cianogênicos os mais importantes, com mais de 25 tipos identificados (VETTER, 2000; CÂMARA; SOTO-BLANCO, 2013). Exemplos destes incluem amigdalina, isolada a partir da família Rosaceae (por exemplo, maçã, pêra, cereja); durrina, presente em plantas do género Sorghum (por exemplo, grãos de sorgo e do capim-Sudão) e linamarina, que é produzido por mandioca, trevo branco e linho (CHEEKE; SHULL, 1985; CHEEKE, 1998; CÂMARA; SOTO-BLANCO, 2013).

A liberação do cianeto contido nos glicosídeos cianogênicos ocorre em duas etapas, através da ação de enzimas endógenas da planta. Primeiramente, a enzima b-glicosidase catalisa a quebra dos hidratos de carbono e as porções cianohidrina ou aglicona. A cianohidrina é então dissociada pela enzima hidroxinitrila-liase a ácido cianídrico, na presença de aldeídos ou cetonas. Na célula de planta intacta, o cianeto não é liberado devido a uma separação espacial entre os glicosídeos cianogênicos, que estão localizados nos vacúolos, e as enzimas que o degradam, que estão localizadas no citosol. Os danos físicos para as células de plantas promovem a combinação dos glicosídeos e as enzimas e a consequente liberação de cianeto (CHEEKE; SHULL, 1985; OSWEILER et al., 1985; CHEEKE, 1998). Portanto, as condições que resultam em danos nas plantas, tais como a secagem, a congelação ou a murchidão, aumentam a toxicidade da planta no momento da ingestão (OSWEILER et al., 1985). Além disso, as enzimas que hidrolisam os glicosídeos cianogênicos são também produzidas 
por bactérias ruminais (MAJAK et al., 1990), sendo encontrada grande variação nas atividades das enzimas hidrolíticas ruminais, com alguns animais que possuem mecanismos mais eficientes para a liberação de cianeto de plantas do que outros (MAJAK et al., 1990; SOTO-BLANCO et al., 2008; SOTO-BLANCO; GÓRNIAK, 2010).

Os fatores que influenciam a toxicidade de plantas cianogênicas são: a concentração do composto na planta, a quantidade de plantas ingerida, a espécie animal, a velocidade de ingestão, os tipos de alimentos ingeridos ao mesmo tempo, a presença de enzimas de degradação ativa em plantas e no trato gastrintestinal, além da capacidade do animal em desintoxicar cianeto (OSWEILER et al., 1985; PÉREZ LÓPEZ et al., 1992). Outro fator que influencia a possível toxicidade destas plantas pode ser a tolerância metabólica, já que animais expostos continuamente a tais compostos podem desenvolver alguma resistência. No entanto, o desenvolvimento desta tolerância foi posta em dúvida (SOTOBLANCO; STEGELMEIER; GÓRNIAK, 2005; SOTO-BLANCO et al., 2008; SOTO-BLANCO; GÓRNIAK, 2010).

O cianeto é prontamente absorvido pelas membranas mucosas e é rapidamente submetido ao processo de detoxificação hepático (SOUSA et al., 2003; CÂMARA; SOTO-BLANCO, 2013). A maior parte do cianeto absorvido (aproximadamente $80 \%$ ) é convertida em tiocianato. Esta reação é catalisada pela enzima rodanase (tiossulfato sulfurtransferase), o que requer tiossulfato como uma fonte de enxofre (BAHRI, 1988; SOUSA et al., 2003; CÂMARA; SOTO-BLANCO, 2013). Uma pequena porção do cianeto absorvida sofre conjugação com a cisteína para formar o ácido 2-imino-4tiazolidina-carboxílico e ácido 2-iminothiazoline4-carboxílico, e com hidroxicobalamina (vitamina $\mathrm{B}_{12}$ ), para formar a cianocobalamina. $\mathrm{O}$ ácido fórmico pode também ser formado em pequenas quantidades. Outra pequena fração do cianeto absorvido é eliminada pelos pulmões sob a forma de HCN (BALLANTYNE, 1987; CÂMARA;
SOTO-BLANCO, 2013). Diante do exposto sobre a alta volatilidade do ácido cianídrico, o exame bioquímico mais usado para verificar sua presença é o teste do papel picrosódico, sendo a metodologia de preparação detalhada nos trabalhos de Carvalho et al. (2011) e Tokarnia et al. (2012).

$\mathrm{O}$ cianeto inibe a ação de enzimas que contêm metais, especialmente ferro, sendo a enzima mais importante inibida denominada citocromo-oxidase, que é uma enzima chave na cadeia respiratória celular (BALLANTYNE, 1987; CÂMARA; SOTOBLANCO, 2013). Ferro trivalente $\left(\mathrm{Fe}^{3+}\right)$ oriundo da enzima citocromo-oxidase reage com complexos de cianeto para formar $\mathrm{CN}$ citocromo-oxidase. Esta reação mantém o ferro na forma trivalente e descarta o sistema de transporte de elétrons da cadeia respiratória, resultando em hipóxia e anóxia citotóxica. Os níveis mais elevados de citocromo-oxidase são encontrados em tecidos com metabolismo oxidativo elevado, tal como o sistema nervoso central e musculatura cardíaca (OSWEILER et al., 1985; VETTER, 2000; CÂMARA; SOTOBLANCO, 2013). Tal bloqueio da cadeia respiratória ao nível da primeira citocromo-oxidase resulta na interrupção da produção de ATP nas mitocôndrias (CÂMARA; SOTO-BLANCO, 2013).

Muitos estudos comprovam o envolvimento do cianeto na produção de radicais livres nos neurônios, resultando em peroxidação lipídica e dano celular (GUNASEKAR; BOROWITZ; ISOM, 1998; YAMAMOTO; TANG, 1998). Além disso, o cianeto inibe tanto a catalase (GUNASEKAR; BOROWITZ; ISOM, 1998) e a enzima glutationa peroxidase (DAVIS; FEAR; WINTON, 1996) . Outros autores sugeriram que o cianeto promove a ativação de N-metil-D-aspartato (NMDA), gerando um fluxo de íons de cálcio, liberação de glutamato e a formação de radicais livres (GUNASEKAR; BOROWITZ; ISOM, 1998; YAMAMOTO; TANG, 1998). Ainda foi observado que o cianeto interfere com diversos neurotransmissores, incluindo $\mathrm{o}$ ácido $\gamma$-aminobutírico (GABA), ácido glutâmico (CASSEL; KARLSSON; SELLSTROM, 1991), a 
acetilcolina, dopamina (CASSEL; KOCH; TIGER, 1995), aminoácidos excitatórios e óxido nítrico (GUNASEKAR; BOROWITZ; ISOM, 1998).

\section{Sinais Clínicos e Achados Patológicos}

A toxicidade aguda ocorre quando as quantidades de cianeto absorvidos excedem grandemente a concentração mínima necessária para inibir a citocromo-oxidase. Além disso, a toxicidade aguda é promovida quando a quantidade de cianeto é maior do que a suportada por enzimas de desintoxicação. Doses elevadas de $\mathrm{HCN}$ podem acarretar a morte do animal em alguns segundos, com convulsões e parada respiratória (TOKARNIA et al., 2012; CÂMARA; SOTO-BLANCO, 2013), enquanto doses não tão elevadas levam o animal ao óbito dentro de minutos a poucas horas após o aparecimento dos primeiros sinais (RIET-CORREA; BEZERRA; MEDEIROS, 2011).

Os sinais clínicos da intoxicação aguda incluem paresia, espasmos musculares generalizados e excitação. Inicialmente, a frequência respiratória é aumentada, seguida por dispneia com abdução dos membros e pescoço estendido, que progride a parada respiratória (OSWEILER et al., 1985; SOTOBLANCO; GÓRNIAK, 2010; NICHOLSON, 2012; CÂMARA; SOTO-BLANCO, 2013). Mucosas cianóticas, nistagmo, tremor de cabeça e pálpebras, seguido de queda, permanência em decúbito lateral com movimentos de pedalagem e opistótono são achados também relatados (TOKARNIA et al., 1999; AMORIM; MEDEIROS; RIET-CORREA, 2005; SOTO-BLANCO; GÓRNIAK, 2010; CÂMARA; SOTO-BLANCO, 2013). Acidose láctica com hiperglicemia ocorre em graus variados, como consequência do metabolismo celular anaeróbico aumentado (BAUD et al., 2002). Ainda é observada, na maioria dos casos, convulsões seguida de morte (OSWEILER et al., 1985; SOTO-BLANCO; GÓRNIAK, 2010; NICHOLSON, 2012).

Nanecropsia,osachadosnãosão patognomônicos. O sangue venoso pode ser vermelho brilhante, pois o oxigênio não é liberado a partir de células vermelhas do sangue para a respiração celular. A coagulação é muitas vezes lenta ou incompleta (MAXIE; YOUSSEF, 2007; NICHOLSON, 2012). Os pulmões e traqueia podem ficar congestos, e pode haver hemorragia subendocárdica e subepicárdica petequial, que sugerem a morte agônica. $\mathrm{O}$ abomaso e intestino podem estar congestos, com a presença de petéquias. O odor característico de cianeto (odor de amêndoa amarga) pode emanar do rúmen (OSWEILER et al., 1985; NICHOLSON, 2012). O exame histológico do cérebro pode revelar lesões degenerativas, principalmente na substância cinzenta ou branca (MAXIE; YOUSSEF, 2007; SOTO-BLANCO; GÓRNIAK, 2010; CÂMARA; SOTO-BLANCO, 2013).

A exposição prolongada ao cianeto tem sido associada a uma série de patologias, incluindo diminuição do crescimento, hipotiroidismo, diabetes pancreática, distúrbios do sistema nervoso e distúrbios reprodutivos (SOTO-BLANCO; STEGELMEIER; GÓRNIAK, 2005; SOTOBLANCO et al., 2008; CÂMARA; SOTOBLANCO, 2013). Além disso, KCN promoveu danos degenerativos moderados a hepatócitos em coelhos (OKOLIE; OSAGIE, 1999), ratos (SOUSA et al., 2002), caprinos (SOTO-BLANCO; STEGELMEIER; GÓRNIAK, 2005; SOTOBLANCO et al., 2008), suínos (MANZANO et al., 2007) e codornas (ROCHA-E-SILVA; CORDEIRO; SOTO-BLANCO, 2010). Sendo também relatada a degeneração das células epiteliais tubulares renais em coelhos (OKOLIE; OSAGIE, 1999), ratos (SOUSA et al., 2002) e suínos (MANZANO et al., 2007).

\section{Principais Plantas Cianogênicas no Nordeste Brasileiro}

Na Tabela 1 estão exemplificados a distribuição dos surtos de intoxicação por plantas cianogênicas no Nordeste brasileiro. 


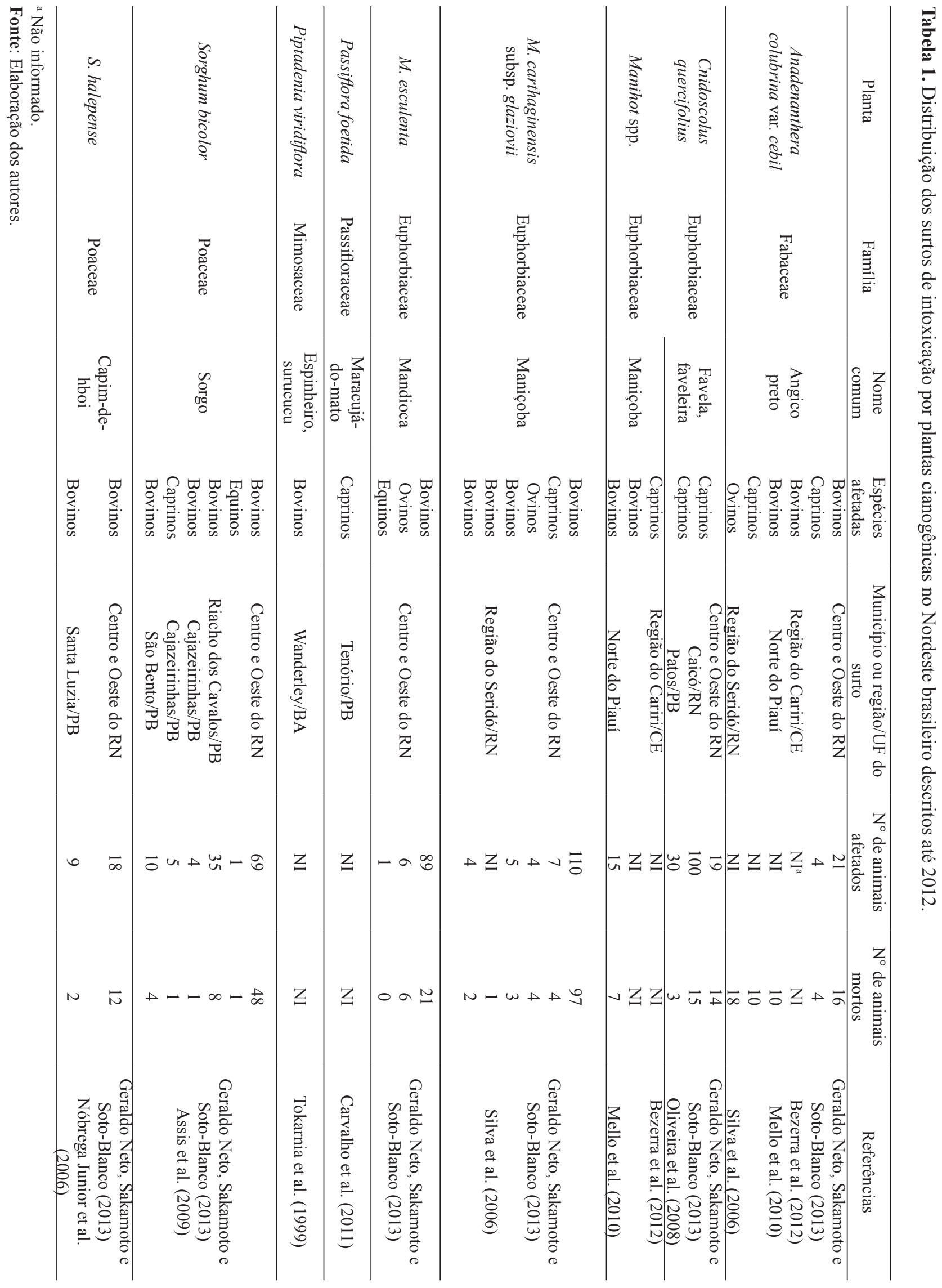


Anadenanthera colubrina var. cebil (Griseb.) Altschul

Esta planta apresenta o nome popular de "angico preto" e possui porte arbóreo. Desde a década de 60 , criadores relatam que as folhas murchas seriam tóxicas para bovinos (TOKARNIA et al., 2012). Dentre os trabalhos de inquéritos epidemiológicos sobre a intoxicação por plantas é unanime dentre os produtores entrevistados que os casos de ingestão da planta ocorrem sempre de forma acidental, geralmente depois de chuvas com ventos fortes ou corte da planta para aproveitamento da madeira (SILVA et al., 2006), quando galhos da árvore se quebram e os animais se alimentam deles (RIETCORREA et al., 2009; MELLO et al., 2010). É notório ainda o discernimento dos produtores com relação à toxicidade da planta para animais de produção, pois o total de $100 \%$ (82/82); 74,6\% $(162 / 217) ; 44,2 \% \quad(27 / 61)$ e $14 \%$ (3/21) dos produtores entrevistados no Seridó, mesorregião Central e Oeste do Rio Grande do Norte (SILVA et al., 2006; GERALDO NETO; SAKAMOTO; SOTO-BLANCO, 2013), Norte Piauiense (MELLO et al., 2010) e Cariri Cearense (BEZERRA et al., 2012), respectivamente, relatam já ter presenciado surtos de intoxicação. Em um dos estudos, o produtor afirma que o angico preto é a principal planta tóxica de sua propriedade (MELLO et al., 2010).

Apenas na década de 90 obteve-se êxito em reproduzir experimentalmente a intoxicação com as folhas frescas da planta provenientes do Estado do Piauí, onde doses de 13,9 g/kg causaram a morte dos cinco bovinos experimentais, enquanto a dose de $10 \mathrm{~g} / \mathrm{kg}$ acarretou a morte de um dos três bovinos amostrados (TOKARNIA; PEIXOTO; DÖBEREINER, 1994). Posteriormente, outros experimentos mostraram a perda gradual de toxicidade das folhas dessecadas ao longo de pouco mais de quatro meses (TOKARNIA et al., 1999). Em estudos utilizando exemplares da planta provenientes da Paraíba, as folhas dessecadas apresentaram-se tóxicas em todas as doses testadas $(5,7,8$ e $10 \mathrm{~g} / \mathrm{kg})$ (AMORIM et al., 2004). Experimentos realizados com amostras coletadas no município de Patos/PB causaram intoxicação por $\mathrm{HCN}$ na dose de $10 \mathrm{mg}$ por kg de peso do animal (MEDEIROS et al., 2000).

\section{Cnidoscolus quecifolius Pohl.}

C. quercifolius (C. phyllacantus), conhecida por favela ou faveleira, é uma planta arbórea, xerófila, lactescente, que possui pelos urticantes. Ocorre em todo o semiárido em regiões de caatinga que envolve áreas dos Estados do Ceará, Rio Grande do Norte, Paraíba, Pernambuco, Alagoas, Sergipe, sudoeste do Piauí, partes do interior da Bahia e do norte de Minas Gerais (RIET-CORREA et al., 2009; TOKARNIA et al., 2012). Estudos recentes mostram que a planta é conhecida pelos produtores no Rio Grande do Norte, já que 94 produtores dos 217 entrevistados declaram saber que a planta é tóxica para ruminantes (GERALDO NETO; SAKAMOTO; SOTO-BLANCO, 2013). Entretanto, nos levantamentos realizados na Paraíba (ASSIS et al., 2009, 2010), Piauí (MELLO et al., 2010) e Ceará (BEZERRA et al., 2012); C. quercifolius não foi mencionada pelos entrevistados (ASSIS et al., 2009, 2010).

Através de numerosos experimentos em caprinos, em que foram utilizadas as folhas frescas, dessecadas, dessecadas trituradas e maduras da favela, em doses que variaram de 0,5 a $4,7 \mathrm{~g} / \mathrm{kg}$, foi comprovada a toxidez dessa planta pela presença de compostos cianogênicos. Todos os 23 caprinos da raça Moxotó apresentaram sinais clínicos semelhantes àqueles observados na intoxicação por $\mathrm{HCN}$, de intensidade leve a acentuada. As folhas frescas causaram intoxicação imediatamente após a administração a partir de $3 \mathrm{~g} / \mathrm{kg}$, enquanto as folhas secas apresentaram toxicidade na mesma dose até após 29 dias de secagem. Já as folhas secas trituradas administradas na dose de $3 \mathrm{~g} / \mathrm{kg}$ ocasionaram sintomatologia clínica leve, e os animais se recuperaram espontaneamente. Outros caprinos experimentais foram submetidos ao tratamento com tiossulfato de sódio a $20 \%$ com recuperação, sendo 
este outro fato associado ao diagnóstico conclusivo de intoxicação por HCN (OLIVEIRA et al., 2008). Ainda acredita-se que a planta possivelmente seja importante em intoxicações em outras espécies de ruminantes (RIET-CORREA et al., 2009).

\section{Manihot spp}

As plantas cianogênicas mais importantes do Brasil pertencem ao gênero Manihot, da família Euphorbiaceae, com distribuição em todo o país, mas com maior predominância do Piauí a Bahia, sendo encontradas nove espécies deste gênero (RIET-CORREA et al., 2009; RIET-CORREA; BEZERRA; MEDEIROS, 2011). A espécie mais conhecida é Manihot esculenta Crantz (mandioca), a "mandioca", porém outras espécies, conhecidas como maniçobas ocorrem em áreas nativas ou são cultivadas como forrageiras (TOKARNIA et al., 2012). A grande maioria dos produtores conhecem a toxicidade e forma de manejo adequado a fim de evitar intoxicações pela planta, entretanto inúmeros surtos são associados à ingestão de Manihot spp. em diversas regiões do semiárido nordestino (ASSIS et al., 2009; MELLO et al., 2010; BEZERRA et al., 2012, GERALDO NETO; SAKAMOTO; SOTOBLANCO, 2013). A intoxicação ocorre quando os animais famintos invadem e ingerem as folhas ricas em glicosídeos cianogênicos desta planta, ou quando as raízes tuberosas são administradas sem o "período de descanso" necessário para a volatilização do HCN (TOKARNIA; DÖBEREINER; VARGAS, 2002; CÂMARA; SOTO-BLANCO, 2013). No Norte do Brasil, existem ainda relatos da intoxicação por manipueira ou tucupi, líquido rico em HCN resultante da compressão da massa ralada das raízes de mandioca (TOKARNIA et al., 2012).

Experimentos com as folhas frescas de duas espécies silvestres (M. piauhyensis e $M$. carthaginensis subsp.glaziovii [M. glaziovii]) causaram a morte de bovinos em doses de 2,5 a $9,3 \mathrm{~g} / \mathrm{kg}$, respectivamente. De forma oposta, as folhas dessecadas entre seis semanas e dez meses não acarretaram sinais clínicos da intoxicação (TOKARNIA; PEIXOTO; DÖBEREINER, 1994). Exemplares da mesma espécie coletados da Paraíba foram ofertadas a bovinos nas doses de 5, 7, 8 e 10g/ $\mathrm{kg}$, ocasionando sinais clínicos em até 20 minutos após a ingestão, enquanto aqueles que receberam a menor quantidade apresentaram apenas sinais clínicos leves durante o movimento (AMORIM et al., 2004). Outro estudo utilizando amostras de M. carthaginensis subsp. glaziovii provenientes do município de Teixeira, Paraíba, demonstraram que a intoxicação ocorre também em caprinos, sendo observado que a planta integra mantém a toxicidade por até 30 dias após a colheita. Entretanto, quando triturada, independente da forma de conservação, era tóxica até 72 horas depois (AMORIM; MEDEIROS; RIET-CORREA, 2005).

\section{Passiflora foetida L.}

$P$. foetida, conhecida vulgarmente como canapúfedorento, maracujá-do-mato ou maracujá-de-estalo, é uma planta trepadeira de crescimento vigoroso e contínuo, com sistema radicular pouco profundo (ANDERSEN; ANDERSEN; JAROSZEWSKI, 1998). Nesta espécie já foram isolados diversos compostos, dentre eles flavonoides, glicosídeos cianogênicos, ácidos graxos e $\alpha$-pírois denominados passifloricinas (DHAWAN; DHAWAN; SHARMA, 2004).

No levantamento de intoxicações por plantas em ruminantes e equídeos no Sertão Paraibano, $P$. foetida é apresentada como uma planta que os produtores relatam como tóxica, mas sem toxicidade comprovada, e associada à ocorrência de abortos em bovinos (ASSIS et al., 2009). Ainda, Passiflora sp. Havia sido testada em estudos anteriores com resultados negativos (TOKARNIA et al., 2012). Ainda em trabalho quantificando as intoxicações por plantas diagnosticadas e estimativa das perdas econômicas (ASSIS et al., 2010), esta espécie sequer é mencionada como causa de mortalidade em animais de produção. Entretanto, recentemente, 
a planta tem sido mencionada em algumas áreas da Paraíba como causadora de intoxicação espontânea, com relatos de um surto no município de Tenório, em caprinos que ingeriram a planta verde, que tinha sido cortada e jogada para os animais (CARVALHO et al., 2011). Enquanto que na região do Seridó do Rio Grande do Norte, alguns proprietários mencionam esta planta como causa de aborto em bovinos, caprinos e ovinos (SILVA et al., 2006).

A fim de determinar a toxicidade de $P$. foetida em caprinos, Carvalho et al. (2011) utilizaram 27 animais experimentais, sendo comprovada que a mesma é uma planta cianogênica causadora de intoxicação após a ingestão das folhas frescas, principalmente no período de estiagem, quando provocou graves sinais clínicos com doses a partir de $8 \mathrm{~g} / \mathrm{kg}$. Este estudo demostra ainda que existe variabilidade da quantidade de glicosídeos cianogênicos de acordo com a época do ano, fato este comprovado pelo teste do papel picrosódico associado a sintomatologia clínica evidenciada nos caprinos intoxicados experimentalmente (CARVALHO et al., 2011).

\section{Piptadenia viridiflora (Kunth) Benth.}

$P$. viridiflora é uma árvore conhecida popularmente como "espinheiro" ou "surucucu", e como o nome popular diz é provida de espinhos. Apesar disso, os bovinos ingerem as folhas desta planta, quando os galhos pendentes ficam ao alcance do gado (TOKARNIA et al., 2012). Existem relatos da intoxicação por $P$. viridiflora no município de Wanderley, região Oeste da Bahia, local este que as folhas foram coletadas para realização de estudo experimental em bovinos. Neste estudo, o quadro clínico-patológico em bovinos foi semelhante ao observado na intoxicação por $A$. colubrina var. cebil, causando a morte do dois bovinos que receberam as folhas recém-coletadas na dose de 10 e $14,1 \mathrm{~g} / \mathrm{kg}$, enquanto que a dosagem de $5 \mathrm{~g} /$ $\mathrm{kg}$ não causou sintomatologia clínica. Outro aspecto importante é que a administração de folhas dessecadas durante 30 e 45 dias nas doses de 4,43 e 7,95 g/kg, respectivamente, continuaram tóxicas (TOKARNIA et al., 1999). Ao contrário do "angico preto", o "espinheiro" não foi citado em nenhum dos trabalhos com levantamento de intoxicações por plantas no Nordeste brasileiro, entretanto acreditase que a epidemiologia da intoxicação seja a mesma (RIET-CORREA et al., 2009).

\section{Sorghum spp}

Sorghum é um gênero com numerosas espécies em regiões tropicais e subtropicais, com diversas espécies usadas como plantas forrageiras (TOKARNIA et al., 2012). Estas plantas podem apresentar altas concentrações de glicosídeos cianogênicos, particularmente quando em fase de brotação, e, na maioria das vezes, com altura menor de $20 \mathrm{~cm}$ ou após 7 semanas do plantio (RIETCORREA et al., 2009). No Nordeste brasileiro, as principais espécies causadoras de quadros de intoxicação são $S$. halepense (capim-de-boi ou sorgo de alepo) (NÓBREGA JUNIOR et al., 2006) e $S$. bicolor (S. vulgare). Não existem relatos comprovadamente provocados por este último, mas nos levantamentos epidemiológicos, S. bicolor é descrito como frequente causa de intoxicação em bovinos e caprinos na região Nordeste (SILVA et al., 2006, ASSIS et al., 2009; BEZERRA et al., 2012; GERALDO NETO; SAKAMOTO; SOTOBLANCO, 2013). Enquanto que na região Sul, a intoxicação por $S$. sudanense (aveia-de-verão ou capim-sudão) apresenta importância relevante na bovinocultura leiteira (JUFFO et al., 2012).

No surto na Paraíba, do total de um rebanho leiteiro de 20 bovinos, nove foram soltos em uma área, ao redor de um açude, onde havia $S$. halepense em estágio de rebrota, com aproximadamente 25-30 $\mathrm{cm}$ de altura. Em um período de aproximadamente 15 minutos após o início da ingestão da planta, os animais apresentaram sinais clínicos compatíveis com intoxicação cianídrica. Dois animais apresentaram sinais mais graves; deitavam-se e 
levantavam-se constantemente evoluindo para uma marcada depressão, decúbito e morte em um período de aproximadamente 3 horas após a ingestão. Os demais bovinos se recuperaram espontaneamente. No município de Currais Novos/RN, plantas em propriedades com históricos de intoxicação foram coletadas e plantadas em vasos. O teste do papel picrosódico resultou em positividade durante todo o seu período vegetativo, sendo que nos primeiros 45 dias a reação ocorreu rapidamente, antes de 1 minuto, e posteriormente, em até 3 minutos (NÓBREGA JUNIOR et al., 2006).

\section{Conclusões}

É notória a grande distribuição e importância da intoxicação por plantas cianogênicas no Nordeste brasileiro, sendo primordial a divulgação dos casos de intoxicação para maior disseminação desta informação nos municípios com pouco conhecimento das espécies causadoras de intoxicação cianídrica, como o Cariri Cearense (BEZERRA et al., 2012). Salienta-se ainda que no diagnóstico diferencial deve-se incluir a intoxicação por outras plantas com evolução superaguda e que também ocorrem na região semiárida, como as intoxicações por Amorimia septentrionalis (Mascagnia rigida), Palicourea aeneofusca (VASCONCELOS et al., 2008), Marsdenia hilariana (PESSOA et al., 2011), M. megalantha (GERALDO NETO, SAKAMOTO; SOTO-BLANCO, 2013) e a intoxicação por nitratos e nitritos (MEDEIROS et al., 2003).

\section{Referências}

AMORIM, S. L.; MEDEIROS, R. M. T.; RIET-CORREA, F. Intoxicação experimental por Manihot glaziovii (Euphorbiaceae) em caprinos. Pesquisa Veterinária Brasileira, Seropédica, v. 25, n. 3, p. 179-187, 2005.

AMORIM, S. L.; MEDEIROS, R. M. T.; RIET-CORREA, F.; OLIVEIRA, A. C. P. Intoxicação experimental com plantas cianogênicas em bovinos. Pesquisa Veterinária Brasileira, Seropédica, v. 24, p. 5-6, 2004. Suplemento.
ANDERSEN, L.; ANDERSEN, A.; JAROSZEWSKI, J. W. Cyanogenesis of Passiflora foetida. Phytochemistry, Oxon, v. 47, n. 6, p. 1049-1050, 1998.

ASSIS, T. S.; MEDEIROS, R. M. T.; RIET-CORREA, F.; ARAÚJO, J. A. S.; DANTAS, A. F. M. Intoxicações por plantas em ruminantes e equídeos no sertão paraibano. Pesquisa Veterinária Brasileira, Seropédica, v. 29, n. 11, p. 919-924, 2009.

ASSIS, T. S.; MEDEIROS, R. M. T.; RIET-CORREA, F.; GALIZA, G. J. N.; DANTAS, A. F. M.; OLIVEIRA, M. D. Intoxicações por plantas diagnosticadas em ruminantes e equinos e estimativa das perdas econômicas na Paraíba. Pesquisa Veterinária Brasileira, Seropédica, v. 30, n. 1, p. 13-20, 2010.

BAHRI, S. A study of detoxification of cyanide into thiocyanate in goats. Penyakit Hewan, Bogor, v. 20, n. 35, p. 34-37, 1988.

BALLANTYNE, B. Toxicology of cyanides. In: BALLANTYNE, B.; MARRS, T. C. (Ed.). Clinical and experimental toxicology of cyanides. Bristol: Wright, 1987. p. 41-126.

BAUD, F. J.; BORRON, S. W.; MÉGARBANE, B.; TROUT, H.; LAPOSTOLLE, F.; VICAUT, E.; DEBRAY, M.; BISMUTH, C. Value of lactic acidosis in the assessment of the severity of acute cyanide poisoning. Critical Care Medicine, Philadelphia, v. 30, n. 9, p. 20442050, 2002.

BEZERRA, C. W. C.; MEDEIROS, R. M. T.; RIVERO, B. R.; DANTAS, A. F. M.; AMARAL, F. R. Plantas tóxicas para ruminantes e equídeos da microrregião do Cariri Cearense. Ciência Rural, Santa Maria, v. 42, n. 6, p. 1070-1076, 2012.

CÂMARA, A. C. L.; SOTO-BLANCO, B. Cyanide poisoning in animals and humans. In: SOTO-BLANCO, B. (Ed.). Cyanide: occurrence, characteristics and applications. Hauppauge: Nova Science Publishers, 2013. p. 23-46.

CARVALHO, F. K. L.; MEDEIROS, R. M. T.; ARAÚJO, J. A. S.; RIET-CORREA, F. Intoxicação experimental por Passiflora foetida (Passifloraceae) em caprinos. Pesquisa Veterinária Brasileira, Seropédica, v. 31, n. 6, p. 477-481, 2011.

CASSEL, G. E.; KOCH, M.; TIGER, G. The effects of cyanide on the extracellular levels of dopamine, 3,4-dihydroxyphenylacetic acid, homovanillic acid, 5-hydroxyindoleacetic acid and inositol phospholipid breakdown in the brain. NeuroToxicology, Amsterdam, v. 16, n. 1, p. 73-82, 1995. 
CASSEL, G.; KARLSSON, L.; SELLSTROM, A. On the inhibition of glutamic acid decarboxylase and gammaaminobutyric acid transaminase by sodium cyanide. Pharmacology \& Toxicology, Copenhagen, v. 69, n. 4, p. 238-241, 1991.

CHEEKE, P. R. Toxins intrinsic to cereal grains and other concentrate feeds. In: Natural toxicants in feeds, forages, and poisonous plants. 2. ed. Danville: Interstate Publishers, 1998. p. 137-162.

CHEEKE, P. R.; SHULL, L. R. Glycosides. In: Natural toxicants in feeds and poisonous plants. Westport: Avi, 1985. p. 173-234.

DANTAS, A. M. C.; SOUZA, D. P. M.; CAVALCANTE, T. V.; ARAÚJO, V. L.; RAMOS, A. T.; MARUO, V. M. Plantas tóxicas de interesse pecuário na região do Ecótono Amazônia e cerrado. Parte I: Bico do Papagaio, Norte do Tocantins. Acta Veterinaria Brasilica, Mossoró, v. 5 , n. 2, p. 178-183, 2011a.

Plantas tóxicas de interesse pecuário na região do Ecótono Amazônia e cerrado. Parte II: Araguaína, Norte do Tocantins. Acta Veterinaria Brasilica, Mossoró, v. 5 , n. 3 , p. $317-324,2011 \mathrm{~b}$.

DAVIS, R. H.; FEAR, J.; WINTON, A. C. Interactions between dietary selenium, copper and sodium nitroprusside, a source of cyanide in growing chicks and laying hens. British Poultry Science, London, v. 37, n. 1, p. 87-94, 1996.

DHAWAN, K.; DHAWAN, S.; SHARMA, A. Passiflora: a review update. Journal of Ethnopharmacology, Limerick, v. 94, n. 1, p. 1-23, 2004.

GERALDO NETO, S. A.; LIMA, J. M.; CÂMARA, A. C. L.; GADELHA, I. V. N.; OLINDA, R. G.; BATISTA, J. S.; SOTO-BLANCO, B. Spontaneous and experimental poisoning by Marsdenia megalantha Goyder \& Morillo in ruminants and a pig. Toxicon, Oxford, v. 63, n. 1, p. 116-119, 2013.

GERALDO NETO, S. A.; SAKAMOTO, S. M.; SOTOBLANCO, B. Inquérito epidemiológico sobre plantas tóxicas das mesorregiões Central e Oeste do Rio Grande do Norte. Ciência Rural, Santa Maria, v. 43, n. 7, p. 1281-1287, 2013.

GUNASEKAR, P. G.; BOROWITZ, J. L.; ISOM, G. E. Cyanide-induced generation of oxidative species: involvement of nitric oxide synthase and cyclooxygenase-2. Journal of Pharmacology and Experimental Therapeutics, Baltimore, v. 285, n. 1, p. 236-241, 1998.

JUFFO, G. D.; PAVARINI, S. P.; WOUTERS, F.; OLIVEIRA, L. G. S.; ANTONIASSI, N. A. B.; CRUZ,
C. E. F.; DRIEMEIER, D. Intoxicação espontânea por Sorghum sudanense em bovinos leiteiros no Rio Grande do Sul. Pesquisa Veterinária Brasileira, Seropédica, v. 32, n. 3, p. 217-220, 2012.

MAJAK, W.; MCDIARMID, R. E.; HALL, J. W.; CHENG, K. J. Factors that determine rates of cyanogenesis in bovine ruminal fluid in vitro. Journal of Animal Science, Champaign, v. 68, n. 6, p. 1648-1655, 1990.

MANZANO, H.; SOUSA, A. B.; SOTO-BLANCO, B.; GUERRA, J. L.; MAIORKA, P. C.; GÓRNIAK, S. L. Effects of long-term cyanide ingestion by pigs. Veterinary Research Communications, Amsterdam, v. 31, n. 1, p. 93-104, 2007.

MAXIE, M. G.; YOUSSEF, S. Nervous system. In: GAXIE, M. G. (Ed.). Jubb, kennedy \& palmer's pathology of domestic animals. 5. ed. Philadelphia: Saunders, 2007. p. 281-457.

MEDEIROS, R. M. T.; NOBRE, V. M. T.; TABOSA, I. M.; RIET-CORREA, F. Toxic plants for ruminants in the state of Paraíba, northeastern Brazil. In: WORLD BUIATRICS CONGRESS, 21., 2000, Montevideo. Proceedings... Montevideo: World Buiatrics Association, 2000. p. 10141-10150.

MEDEIROS, R. M. T.; RIET-CORREA, F.; TABOSA, I. M.; SILVA, Z. A.; BARBOSA, R. C.; MARQUES, A. V. M. S.; NOGUEIRA, F. R. B. Intoxicação por nitratos e nitritos em bovinos por ingestão de Ehinochloa polystachya (capim-mandante) e Pennisetum purpureum (capim-elefante) no sertão da Paraíba. Pesquisa Veterinária Brasileira, Seropédica, v. 23, n. 1, p. 17-20, 2003.

MELLO, G. W. S.; OLIVEIRA, D. M.; CARVALHO, C. J. S.; PIRES, L. V.; COSTA, F. A. L.; RIET-CORREA, F.; SILVA, S. M. M. Plantas tóxicas para ruminantes e equídeos no Norte Piauiense. Pesquisa Veterinária Brasileira, Seropédica, v. 30, n. 1, p. 1-9, 2010.

NICHOLSON, S. S. Cyanogenic plants. In: GUPTA, R. C. (Ed.). Veterinary toxicology. 2. ed. London: Academic Press, 2012. p. 1113-1116.

NÓBREGA JUNIOR, J. E.; RIET-CORREA, F.; MEDEIROS, R. M. T.; DANTAS, A. F. M. Intoxicação por Sorghum halepense em bovinos no semiárido. Pesquisa Veterinária Brasileira, Seropédica, v. 26, n. 4, p. 201-204, 2006.

OKOLIE, N. P.; OSAGIE, A. U. Liver and kidney lesions and associated enzyme changes induced in rabbits by chronic cyanide exposure. Food and Chemical Toxicology, Oxford, v. 37, n. 7, p. 745-750, 1999. 
OLIVEIRA, D. M.; PIMENTEL, L. A.; ARAÚJO, J. A. S.; MEDEIROS, R. M. T.; DANTAS, A. F. M.; RIETCORREA, F. Intoxicação por Cnidoscolus phyllacanthus (Euphorbiaceae) em caprinos. Pesquisa Veterinária Brasileira, Seropédica, v. 28, n. 4, p. 36-42, 2008.

OSWEILER, G. D.; CARSON, T. L.; BUCK, W. B.; VAN GELDER, G. A. Cyanide and cyanogenic plants. In: _. (Ed.). Clinical and diagnostic veterinary toxicology. 3. ed. Dubuque: Kendall/Hunt, 1985. p. 455459.

PÉREZ LÓPEZ, J. A.; MARTÍNEZ, F. G.; MARTÍNEZ, R. R.; HORTA R., J. M. Variación del contenido de cianuro en Prunus brachybotrya en la segunda mitad de su desarrollo. Veterinaria México, México, v. 23, n. 2, p. 131-133, 1992.

PESSOA, C. R. M.; RIET-CORREA, F.; MEDEIROS, R. M. T.; SIMÕES, S. V. D.; RAPINI, A. Poisoning by Marsdenia hilariana and Marsdenia megalantha (Apocynaceae) in ruminants. Toxicon, Oxford, v. 58, n. 6-7, p. 610-613, 2011.

RIET-CORREA, F.; BEZERRA, C. W. C.; MEDEIROS, R. M. T. Plantas tóxicas do Nordeste. Patos: Sociedade Vicente Palloti, 2011. 82 p.

RIET-CORREA, F.; MEDEIROS, R. M. T.; PFISTER, J.; SCHILD, A. L.; DANTAS, A. F. M. Cyanogenic plants. In: . (Ed.). Poisonings by plants, mycotoxins and related substances in brazilian livestock. Patos: Sociedade Vicente Palloti, 2009. p. 149-157.

ROCHA-E-SILVA, R. C.; CORDEIRO, L. A. V.; SOTOBLANCO, B. Cyanide toxicity and interference with diet selection in quail (Coturnix coturnix). Comparative Biochemistry and Physiology C, Toxicology \& Pharmacology, New York, v. 151, n. 3, p. 294-297, 2010.

SCHONS, S. V.; LOPES, T. V.; MELO, T. L.; LIMA, J. P.; RIET-CORREA, F.; BARROS, M. A. B.; SCHILD, A. L. P. Intoxicações por plantas em ruminantes e equídeos na região central de Rondônia. Ciência Rural, Santa Maria, v. 42, n. 7, p. 1257-1263, 2012.

SILVA, D. M.; RIET-CORREA, F.; MEDEIROS, R. M. T.; OLIVEIRA, O. F. Plantas tóxicas para ruminantes e equinos no Seridó Ocidental e Oriental do Rio Grande do Norte. Pesquisa Veterinária Brasileira, Seropédica, v. 26, n. 4, p. 223-236, 2006.

SOTO-BLANCO, B.; GÓRNIAK, S. L. Toxic effects of prolonged administration of leaves of cassava (Manihot esculenta Crantz) to goats. Experimental and Toxicologic Pathology, Jena, v. 62, n. 4, p. 361-366, 2010.

SOTO-BLANCO, B.; STEGELMEIER, B. L.; GÓRNIAK, S. L. Clinical and pathological effects of short-term cyanide repeated dosing to goats. Journal of Applied Toxicology, Philadelphia, v. 25, n. 6, p. 445-450, 2005.

SOTO-BLANCO, B.; STEGELMEIER, B. L.; PFISTER, J. A.; GARDNER, D. R.; PANTER, K. E. Comparative effects of prolonged administration of cyanide, thiocyanate and chokecherry (Prunus virginiana) to goats. Journal of Applied Toxicology, Philadelphia, v. 28, n. 3, p. 356-363, 2008.

SOUSA, A. B.; MANZANO, H.; SOTO-BLANCO, B.; GÓRNIAK, S. L. Toxicokinetics of cyanide in rats, pigs and goats after oral dosing with potassium cyanide. Archives of Toxicology, Berlin, v. 77, n. 6, p. 330-334, 2003.

SOUSA, A. B.; SOTO-BLANCO, B.; GUERRA, J. L.; KIMURA, E. T.; GÓRNIAK, S. L. Does prolonged oral exposure to cyanide promote hepatotoxicity and nephrotoxicity? Toxicology, Amsterdam, v. 174, n. 2, p. 87-95, 2002.

TOKARNIA, C. H.; BRITO, M. F.; BARBOSA, J. D.; VARGAS, P. V.; DÖBEREINER, J. Plantas cianogênicas. In: . (Ed.). Plantas tóxicas do Brasil para animais de produção. 2. ed. Rio de Janeiro: Helianthus, 2012. p. 443-460.

TOKARNIA, C. H.; DÖBEREINER, J.; VARGAS, P. V. Poisonous plants affecting livestock in Brazil. Toxicon, Oxford, v. 40, n. 12, p. 1635-1660, 2002.

TOKARNIA, C. H.; PEIXOTO, P. V.; BRITO, M. F.; DUARTE, M. D.; BRUST, L. A. C. Estudos experimentais com plantas cianogênicas em bovinos. Pesquisa Veterinária Brasileira, Seropédica, v. 19, n. 2, p. 84-90, 1999.

TOKARNIA, C. H.; PEIXOTO, P. V.; DÖBEREINER, J. Intoxicação experimental por Piptadenia macrocarpa (Leg. Mimosoideae) em bovinos. Pesquisa Veterinária Brasileira, Seropédica, v. 14, n. 2-3, p. 57-63, 1994.

VASCONCELOS, J. S.; RIET-CORREA, F.; DANTAS, A. F.; MEDEIROS, R. M. T.; DANTAS, A. J. A. Mortes súbitas em bovinos causadas por Palicourea aeneofusca (Rubiaceae) e Mascagnia rígida (Malpighiaceae) na Zona da Mata Paraibana. Pesquisa Veterinária Brasileira, Seropédica, v. 28, n. 10, p. 457-460, 2008.

VETTER, J. Plant cyanogenic glycosides. Toxicon, Oxford, v. 38, n. 1, p. 11-36, 2000.

YAMAMOTO, H. A.; TANG, H. W. Effects of 2-amino7-phosphonoheptanoic acid, melatonin or NG-nitro-Larginine on cyanide or N-methyl-D-aspartate-induced neurotoxicity in rat cortical cells. Toxicology Letters, Amsterdam, v. 94, n. 1, p. 13-18, 1998. 
